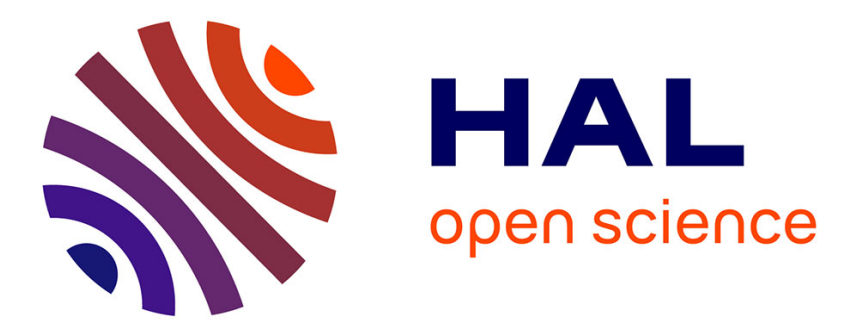

\title{
L'artisanat dans la Délos hellénistique: essai de bilan archéologique
}

Michèle Brunet

\section{To cite this version:}

Michèle Brunet. L'artisanat dans la Délos hellénistique: essai de bilan archéologique. Topoi Orient Occident, 1998, 8/2, pp. 681- 691. 10.3406/topoi.1998.1781 . hal-01532789

\section{HAL Id: hal-01532789 \\ https://hal.science/hal-01532789}

Submitted on 3 Jun 2017

HAL is a multi-disciplinary open access archive for the deposit and dissemination of scientific research documents, whether they are published or not. The documents may come from teaching and research institutions in France or abroad, or from public or private research centers.
L'archive ouverte pluridisciplinaire HAL, est destinée au dépôt et à la diffusion de documents scientifiques de niveau recherche, publiés ou non, émanant des établissements d'enseignement et de recherche français ou étrangers, des laboratoires publics ou privés.

\section{(1) (1) $\$$}

Distributed under a Creative Commons Attribution - NonCommercial - NoDerivatives| 4.0 


\section{L'artisanat dans la Délos hellénistique : essai de bilan} archéologique

Michèle Brunet

Citer ce document / Cite this document :

Brunet Michèle. L'artisanat dans la Délos hellénistique : essai de bilan archéologique. In: Topoi, volume 8/2, 1998. pp. 681691 ;

doi : 10.3406/topoi.1998.1781

http://www.persee.fr/doc/topoi_1161-9473_1998_num_8_2_1781

Document généré le 21/05/2016 


\section{L'ARTISANAT DANS LA DÉLOS HELLÉNISTIQUE : ESSAI DE BILAN ARCHÉOLOGIQUE}

Recenser, à l'échelle de la totalité d'une cité antique et pour une période donnée, l'ensemble des artisanats attestés par des vestiges et en étudier la distribution spatiale, afin de mettre en lumière les logiques qui ont présidé à leur implantation : le projet apparaît a priori d'autant plus séduisant qu'il s'avère relativement inédit.

\section{QUESTIONS DE MÉTHODE}

Bien que la Délos hellénistique soit à bien des égards une cité exceptionnelle et ne puisse donc illustrer une quelconque norme qui serail valable pour l'ensemble des cités grecques, le choix de cet exemple s'impose sur le plan documentaire. L'ampleur de l'exploration archéologique du site, quasi continue depuis plus d'un siècle, les prospections récentes du territoire, à Délos comme à Rhénée, enfin, la relative abondance des publications traitant des productions artisanales déliennes ${ }^{1}$ font de Délos un cas tout à fait privilégié dans la perspective de notre enquête. Toutefois, il ne faut pas oublier qu'à l'heure actuelle plus de la moitié de la ville est encore sous terre et que, malgré le dépeuplement amorcé au I ${ }^{\mathrm{er}} \mathrm{s}$. av. J.-C., qui figea une partie de la ville dans son état hellénistique, la continuité de l'occupation jusqu'au $\mathrm{VII}^{\mathrm{e}} \mathrm{s}$. de notre ère entraîna inévitablement le démantèlement d'installations artisanales antérieures. Enfin, le caractère parfois « expéditif » (en regard des exigences qui sont les nôtres) des grandes fouilles fut certainement à l'origine d'une perte d'informations, dans des proportions qu'il est cependant impossible d'apprécier. Tout en étant très honorable par comparaison

1. On les trouvera recensées dans la bibliographie raisonnée en fin d'article. Pour la localisation des Quartiers et des monuments mentionnés, on se reportrera aux cartes et plans du Guide de Délos ${ }^{3}$ (1983) et de l'ouvrage collectif Délos, île sacrée et ville cosmopolite (1996). 
avec d'autres cités (que l'on songe à Athènes...), le bilan archéologique concernant l'artisanat délien ne saurait donc qu'être, d'entrée de jeu, partiel.

Mais, de façon plus gênante, une enquête menée indépendamment des textes court le risque de livrer une image à la fois floue et déformée de l'artisanat délien. Truisme ? On me pardonnera d'insister pour commencer sur quelques points de méthode, car ils éclairent la teneur des résultats obtenus et conditionnent la validité de cette étude.

La réunion d'un corpus de vestiges témoins d'activités artisanales n'est pas une opération aussi simple qu'il n'y paraît ; elle se heurte à un certain nombre d'impedimenta qui sont autant de révélateurs d'une difficulté de fond : comment, en se fondant sur des vestiges matériels, appréhender l'« artisanat », c'est-à-dire une catégorie socio-économique complexe dont les contours, dans le contexte de la société grecque antique, sont loin d'être clairement délimités ${ }^{2}$ ?

Que l'on tente de définir cette notion en s'appuyant sur le vocabulaire et sur les textes antiques ou que l'on adopte une définition actuelle du terme ${ }^{3}$, l'artisanat englobe à l'évidence une grande variété d'activités de fabrication professionnalisées, de métiers spécialisés qui sont pratiqués à des fins commerciales. C'est précisément cette vocation commerciale qui permet de différencier l'artisanat proprement dit de ce que l'on nomme souvent de manière très ambiguë $l^{\prime}$ '« artisanat domestique », domaine qui inclut toutes ces activités de fabrication - notamment celle de vêtements ou de denrées alimentaires, farine, huile et vin - qui, chez les Grecs de l'antiquité, avaient pour cadre l'oikos et qui visaient essentiellement à satisfaire les besoins privés. Nonobstant leur importance, on doit exclure ces activités de notre inventaire si l'on veut conserver un sens aux mots et à l'étude un objet cohérent : en effet, renoncer à distinguer entre les deux sphères de production conduirait inévitablement à faire de notre corpus un fourre-tout compilant toutes les traces d'un processus matériel de fabrication. A ce titre, presque chaque maison délienne passerait alors pour avoir été le siège d'un artisanat, sous prétexte que quelques pesons, pigments ou un moulin à tré-

2. Cf. ici-même les analyses de V. CHANKOWSKI, N. MASSAR et D. VIVIERS.

3. Comme par exemple celle que proposent des géographes : « activité productive pratiquée par des entrepreneurs indépendants, sans salariés ou avec un petit nombre de salariés (...) éventuellement associée à une commercialisation au détail. L'artisanat comprend la production d'objets, de denrées, l'installation de machines ou équipements, la prestation de services. Exclut en France les professions libérales et les commerces de détail non associés à un processus de transformation. L'artisan a un atelier, une boutique ou travaille à domicile chez le client », s.v. « artisanat », R. BRUNET, R. FERRAS, H. THÉRY, Les mots de la géographie 3 (1993). 
mie y auraient été découverts ${ }^{4}$. Nécessaire, la distinction n'a malheureusement rien d'évident au regard des vestiges. Certes, la typologie des appareils, la configuration des installations et leur situation fournissent quelques éléments discriminants quant à la visée commerciale ou non de la fabrication, mais ces critères combinés présenteront toujours un caractère incertain et contestable. Il y a donc là une vraie difficulté dont il faut prendre la mesure car, au-delà du cas délien ici en cause, l'équivoque n'est pas circonscrite à un secteur qui pourrait paraître marginal ; une incertitude identique entoure, par exemple, le statut des ateliers de fabrication d'amphores. A Thasos où l'on en connaît aujourd'hui une vingtaine, ce ne sont ni les vestiges de fours ni les dépotoirs ni même leur implantation à plus ou moins grande proximité des fermes qui permettent de distinguer entre les fabrications véritablement artisanales et les productions «appendices de la vie agricole », couvrant les besoins particuliers d'une exploitation 5 .

Second écueil à souligner avant d'entrer dans le vif du sujet : inévitablement, un corpus de vestiges ne nous livrera qu'une image très sélective de l'artisanat délien. Recensant uniquement les activités artisanales susceptibles de laisser des traces sous la forme d'équipements immobiliers ou mobiliers, de matière première et de rebuts de fabrication qui se concentrent dans des ateliersboutiques, l'inventaire conduit à restreindre de manière indue l'artisanat antique au seul domaine de la fabrication d'objets, utilitaires ou d'art, et laisse dans l'ombre bien d'autres secteurs de la production, « invisibles » sur le terrain. Ainsi potiers, sculpteurs, orfèvres ou verriers sont-ils toujours les « héros » incontestés de l'artisanat grec, tailleurs de pierre et maçons jouant à l'occasion les seconds rôles. En dépit d'une indéniable originalité, l'inventaire délien ne fait que confirmer cette tendance : restons conscients que cette vision partiale ne reflète que bien peu la complexité historique de l'artisanat.

\section{BILAN ARCHÉOLOGIQUE}

\subsection{Activités et implantations}

Après sélection des vestiges conformément aux critères évoqués ci-dessus, il devient possible d'établir une double liste, des activités artisanales et des lieux où elles se trouvent attestées. Il m'a semblé plus commode de présenter ce bilan sous la forme d'un tableau, où sont distingués deux groupes principaux : le premier, le plus fourni et diversifié, rassemble les activités de production d'objets ou biens plus ou moins luxueux, le second deux activités bien plus vernaculaires, la fabrication artisanale de farine et d'huile. La présence de sculpteurs, toreutes,

4. Cf. le tableau des « occupations commerciales et industrielles des Déliens » dressé par DEONNA (1948), qui renonce d'emblée à faire la distinction entre « les industries domestiques et les industries commerciales » .

5. Cf. à ce sujet la communication d'Y. GARLAN, p. 581-590. 
parfumeurs, verriers et teinturiers se conçoit parfaitement dans une cité où résidait une population de commerçants aisés et qui abritait un sanctuaire très fréquenté, de même que les meuneries et huileries visaient à satisfaire les besoins en produits alimentaires courants d'une population nombreuse, à une époque où les agriculteurs pratiquant l'autoconsommation étaient sans nul doute minoritaires. Bien qu'elles n'aient encore fait l'objet d'aucune étude d'ensemble, les petites carrières disséminées dans toute l'île (et sans doute à Rhénée également) ne doivent pas être oubliées : elles sont à leur manière, en dehors des ruines elles-mêmes, les seuls témoins matériels d'une des activités artisanales des plus importantes dans toute cité et, malgré leurs dimensions réduites à Délos, elles attestent une exploitation systématique des pierres locales pour la construction, tant privée que publique.

\begin{tabular}{|c|c|}
\hline \multicolumn{2}{|r|}{ ARTISANATS D'ART ET DE PRODUITS DE LUXE } \\
\hline $\begin{array}{l}\text { ATELIERS DE } \\
\text { SCULPTEURS } \\
\text { (Marbre) }\end{array}$ & $\begin{array}{l}\text { - Boutiques } 103 \text { et } 106 \text { à l'angle SO de l'Agora des Italiens, GD } 52 . \\
\text { - Maison de Kerdon, GD } 83 \text {. } \\
\text { - Portique de Philippe, GD } 3 .\end{array}$ \\
\hline TOREUTIQUE & $\begin{array}{l}\text { - Moules rassemblés dans une boutique au N. de l'Ilot des Bronzes, } \\
\text { GD } 59 \mathrm{C} \text {, l'emplacement originel de l'atelier est inconnu. }\end{array}$ \\
\hline TEINTURERIES & $\begin{array}{l}\text { - Extrémité } \mathrm{N} \text { du Quartier du stade } G D 79.1 \text {, près du rivage. } \\
\text { - Plaine à l'E. de la ville, près du rivage, } 500 \mathrm{~m} \text { au } \mathrm{S} \text {. de la } \\
\text { Synagogue, } G D 80.1 \text {. } \\
\text { - Au centre de la baie de Fourni, près du rivage. }\end{array}$ \\
\hline PARFUMERIES & $\begin{array}{l}\text { - Maison B du Quartier du Stade, GD 79, deux maies et quatre fours } \\
\text { in situ. } \\
\text { - Palestre de granit, GD } 66 \text { une maie in situ. } \\
\text { - Maisons à l'O. de la Salle hypostyle, GD } 50 \text { (maies déplacées). } \\
\text { - Maisons sur la bordure E. de l'Agora des Compétaliastes, GD } 2 \\
\text { (maie déplacée). } \\
\text { - Quartier du théâtre, Maison du Dionysos GD } 120 \text { (maie déplacée). } \\
\text { - Quartier du théâtre, Maison du Trident, GD } 118 \text { (maie déplacée). }\end{array}$ \\
\hline $\begin{array}{l}\text { ATELIERS DE } \\
\text { VERRIERS }\end{array}$ & $\begin{array}{l}\text { - Magasins à l'O. de la Maison aux stucs, } G D 87 . \\
\text { - Zone au S. du Samothrakeion, GD 93. } \\
\text { - Etablissement dans la haute vallée de l'Inopos }\end{array}$ \\
\hline $\begin{array}{l}\text { FABRIQUES } \\
\text { D'AULOI }\end{array}$ & $\begin{array}{l}\text { - Boutique voisine au N. de la Maison de Kerdon, GD } 83 . \\
\text { - Rez-de chaussée du Monument de granit, GD } 54 .\end{array}$ \\
\hline $\begin{array}{l}\text { ATELIERS DE } \\
\text { COROPLATHES }\end{array}$ & $\begin{array}{l}\text { - Boutique sur la bordure S. de l'Agora des Italiens, GD } 52 . \\
\text { - Quartier du théâtre, insula VI, maison B. }\end{array}$ \\
\hline
\end{tabular}




\begin{tabular}{|l|l|}
\hline \multicolumn{2}{|c|}{ ARTISANATS LIÉS À L'ALIMENTATION } \\
\hline HUILERIES & $\begin{array}{l}\text { - Quartier du théâtre, Insula III, maison O, maie et contrepoids in } \\
\text { situ } \\
\text { - Quartier du théâtre, Insula III, maison T (maie déplacée) } \\
\text { - Maison au S. de l'Établissement des Poseidoniastes, GD } 57 \text { (maie } \\
\text { déplacée) }\end{array}$ \\
\hline MEUNERIES & $\begin{array}{l}\text { - Maison des sceaux, GD } 59 \mathrm{D}, \text { moulin rotatif in situ. } \\
\text { Emplacements au sol d'un moulin rotatif: } \\
\text { - Maison de Kerdon, GD 83. } \\
\text { - Maison sur la bordure E. de l'Agora des Compétaliastes, GD 2. } \\
\text { - Quartier du thêâtre, Rue du théâtre, boutiques 26, 27 b, 33, } 41 \text { b. } \\
\text { - Quartier du thêâtre, Rue 5, boutique 10. } \\
\text { - Quartier du thêâtre, Rue supérieure du théâtre, boutique 7. } \\
\text { - Ferme aux jambages de granit au centre de l'île. }\end{array}$ \\
\hline
\end{tabular}

\begin{tabular}{|l|l|}
\hline CARRIÈRES & $\begin{array}{l}\text { - Carrières de marbre au SE du Cynthe et près du théâtre. } \\
\text { - Petites carrières de granite dispersées dans toute la moitié S. } \\
\text { - Petites carrières de gneiss sur les presqu'îles N. }\end{array}$ \\
\hline
\end{tabular}

Le lecteur se reportera à la bibliographie en fin d'article et à certaines des communications réunies dans ce volume pour le détail des découvertes concernant chaque artisanat; je me contenterai pour ma part de mettre l'accent brièvement sur les découvertes les plus récentes, qui ont révélé l'existence de parfumeries, de meuneries et d'huileries à Délos.

\subsection{La parfumerie du Quartier du Stade}

Un nettoyage effectué par J.-P. Brun et moi-même au printemps 97 dans la maison B du Quartier du stade, dégagée par A. Plassart en 1913, est venu pleinement corroborer un passage de Pline l'Ancien (HN, XIII,4) évoquant une production de parfums réputés dans la Délos hellénistique. En effet, en contrebas de quatre fours qui avaient fait interpréter cette demeure comme une auberge (voir $G D$ 79), nous avons mis au jour les restes du massif de fondation de deux pressoirs juxtaposés du type dit « à coins », semblables à celui représenté sur la peinture des «Amours parfumeurs » de la Maison des Vettii à Pompéi. Ces appareils permettaient la production de petites quantités d'huile, qui étaient ensuite mélangées à des substances odorantes au cours d'un chauffage au bain-marie, d'où la présence des fours aux côtés des deux maies découvertes renversées au moment de la fouille. Cette parfumerie fonctionna à la fin du $\mathrm{II}^{\mathrm{e}}$ et au début du $\mathrm{I}^{\mathrm{er}}$ s. av. J.-C., puis fut démantelée pour faire place à ce qui doit être un établissement de bains. Ce laboratoire faisait sans nul doute également office de boutique : la fabrication du parfum s'effectuait sous les yeux de la clientèle, ce qui doit expliquer pourquoi les maies de marbre sont d'une facture si soignée. Plus petites que les maies utilisées pour la fabrication des huiles alimentaires, qui ne sont que sommairement dégrossies, leurs faces sont parfaitement dressées et 
un motif de volutes autour d'un cœur en relief entoure leur bec verseur. Or, sur les douze maies repérées dans les ruines déliennes, huit présentent ce décor raffiné : cette proportion des $2 / 3$ de l'échantillon parvenu jusqu'à nous est sans doute l'indice d'une production de parfums effectivement développée à l'époque hellénistique.

\subsection{L'huilerie du Quartier du théâtre}

Au cours de la même campagne du printemps 97, J.-P. Brun et moi-même avons repris l'étude de l'huilerie située dans le bas du Quartier du théâtre, maison O de l'insula III, dégagée par Mayence en 1906. Quelques sondages nous ont permis d'établir de façon définitive que cet établissement était bien destiné à la fabrication d'huile, de restituer le fonctionnement exact des pressoirs et de fixer la chronologie des installations. Dans une maison dont la construction remonte au $V^{e} s$., furent mis en place au cours du premier quart du Ier $^{e}$. deux pressoirs du type à contrepoids et six pithoi identiques, d'une capacité respective de 6501 . L'huilerie fonctionna peu de temps, jusqu'au milieu du $\mathrm{I}^{\text {er }}$ s., et le local abandonné ne fut pas réoccupé par la suite. La présence des deux pressoirs se justifie en relation avec les procédures d'extraction d'huiles de qualités différentes. L'un servait très vraisemblablement à l'extraction de l'huile de première pression, la plus pure, destinée à la consommation, tandis que l'autre, plus puissant et associé à des réceptacles de plus grande capacité, était utilisé pour les huiles de deuxième et troisième pression, obtenues par adjonction d'eau chaude et réservées à des usages non alimentaires. Les analyses morphométriques des noyaux d'olives récupérés dans les fonds de pithoi indiquent qu'ils appartiennent à une variété très proche de l'actuclle olive grecque Koroneiki. En rapprochant les rendements de cette variété d'oliviers de la capacité totale de stockage de l'huilerie (3900 1), on peut estimer que cet établissement traitait la récolte annuelle de 650 oliviers au maximum. Ce chiffre - qu'il faut prendre comme un ordre de grandeur - confirme à mon sens le caractère artisanal de l'huilerie, que suggérait déjà par ailleurs son implantation en plein cœur de la ville, car aucun exploitant ne pouvait posséder, ni à Délos ni à Rhénée, autant d'oliviers : à l'époque de l'Indépendance, les inventaires des domaines d'Apollon n'en dénombrent aucun sur les deux îles ${ }^{6}$.

\subsection{Meuneries}

Dans un article paru dernièrement, j'ai proposé une restitution du fonctionnement d'un moulin à céréales rotatif, très original tant du point de vue de sa conception que de sa réalisation en pièces détachées. Cet appareil de mouture, qui me paraît attesté à Délos par les inscriptions dès le III ${ }^{\mathrm{e}} \mathrm{s}$., devait être

6. Seuls les deux domaines de Mykonos en possèdent quelques-uns ; ce sont d'ailleurs majoritairement des oléastres, qui fournissent une huile recommandée pour la fabrication du parfum. 
assez répandu à l'époque hellénistique, si l'on en juge par le nombre de fragments dispersés dans les ruines ou remployés comme moellons dans les murs de maisons. A Pompéi comme à Ostie, des boulangeries ont été identifiées grâce à la découverte de gros moulins rotatifs (moulins dits « pompéiens »), dont l'utilisation est clairement artisanale compte tenu de leurs capacités de mouture. Or ce modèle d'appareil n'est représenté à Délos que par des exemplaires de dimensions très réduites, et toutes les autres catégories de moulins attestées en concomitance ne peuvent avoir eu qu'un usage domestique. Le moulin rotatif en pièces détachées apparaît donc localement comme le seul équivalent du moulin pompéien en regard de ses capacités de mouture importantes. Cette hypothèse se voit confortée par le fait que l'on observe dans plusieurs locaux bordant les rues du Quartier du théâtre un aménagement du dallage similaire à celui qui fut constaté autour de l'unique moulin découvert en place, dans la Maison des sceaux. Sur la foi du parallélisme avec les installations pompéiennes, ces boutiques ont été interprétées par Chamonard et Deonna comme des boulangeries ; il me semble plus juste d'y voir de simples meuneries, puisqu'elles ne possédaient aucun four.

\section{ESQUISSE D'ANALYSES}

La distribution spatiale des ateliers déliens se révèle peu significative : parmi les secteurs de la ville qui sont dégagés, aucun n'a, semble-t-il, une vocation plus particulièrement artisanale. Les locaux abritant une activité artisanale se répartissent un peu partout dans l'agglomération ou sur ses franges, certains se trouvent à l'intérieur de « maisons » 7 , d'autres, plus nombreux, sont des pièces indépendantes dont on admet qu'elles faisaient également office de boutiques. La plupart de ces ateliers se situent tout naturellement en bordure de voies de circulation, soit dans les divers quartiers d'habitation, soit dans des zones publiques, abords du sanctuaire, agoras.

Malgré l'étroitesse du corpus analysé, la répartition des différents artisanats paraît donc obéir principalement à une logique d'ordre commercial, privilégiant la proximité avec la clientèle potentielle. Les meuneries comme les huileries se trouvent dans les quartiers d'habitation, les fabricants de statuettes en marbre et l'atelier de coroplathes sont installés aux abords du sanctuaire d'Apollon, tandis que les parfumeurs se rencontrent près des établissements sportifs, dans le Quartier du stade et à la Palestre de granit, mais aussi en ville. Seuls les teinturiers ont été contraints à une situation excentrée, en limite d'agglomération et en bord de

7. Il ne faut pas toutefois se laisser abuser par cette dénomination, commode pour les fouilleurs : elle ne préjuge en rien de l'utilisation effective qui était faite des locaux. C'est ainsi que la présence d'activités artisanales devrait conduire à s'interroger plus avant sur la «Maison de Kerdon » ou sur le rez-de-chaussée de la «Maison des sceaux ». 
mer, à la fois pour des raisons liées à leur approvisionnement en matière première et pour éviter les nuisances olfactives.

Enfin, ce petit corpus révèle une absence flagrante : aucun atelier de potier digne de ce nom n'a jamais été repéré, ni en ville ni dans la campagne. Est-ce là une réalité ou un effet de l'insuffisance des fouilles et des prospections ? $\mathrm{Si}$, de fait, il ne se trouve aucun gisement naturel d'argile à Délos même (où j'ai la certitude qu'il n'y a aucun dépotoir céramique dans le territoire), il conviendrait d'avoir les mêmes assurances concernant Rhénée, avant de conclure que l'on importait à Délos la totalité de la vaisselle, notamment commune.

On aura constaté qu'il n'a point été question des pressoirs à vin étudiés par $\mathrm{Ph}$. Bruneau et $\mathrm{Ph}$. Fraisse. Datés des époques impériale et paléochrétienne, ils sortent de la période retenue pour cette enquête, mais cette omission traduit surtout mes hésitations quant à leur usage dans un cadre artisanal ou purement domestique. L'apparition à date tardive de ces pressoirs implantés dans les ruines de la ville hellénistique - l'un bouche une rue du Quartier du théâtre, l'autre s'installe dans un bâtiment public abandonné - témoigne en premier lieu d'une évolution technologique dans la manière de fabriquer le vin, qui est générale à l'échelle de la Méditerranée, mais principalement d'une ruralisation de la « ville », où se concentre dorénavant une communauté réduite d'agriculteurs. Précédemment, c'était sans doute dans chacune des fermes du territoire que le produit des vignobles peu étendus était traité, avec des moyens plus rudimentaires. Désormais, cette activité de transformation des vendanges s'effectue dans le bourg agricole, sans qu'il s'agisse pour autant d'une production à une échelle artisanale.

Cet inventaire rapide des artisanats attestés par l'archéologie dans la Délos hellénistique souligne le caractère hors du commun d'une ville opulente où s'épanouissaient, parallèlement à des productions communes à la plupart des cités, des artisanats d'exception pour une clientèle à demeure ou de passage. Certains d'entre eux font même figure de spécialités locales, à en croire les sources textuelles qui les signalent comme « déliennes ». La liste de ces spécialités peut aujourd'hui être étendue à des domaines plus modestes ; de fait, en l'état actuel de la documentation, Délos est la seule ville grecque d'époque hellénistique où ont été identifiées sur le terrain une huilerie artisanale et des meuneries équipées d'appareils originaux et fort peu diffusés hors de ses parages immédiats. Seules les villes romaines offrent des parallèles pour cette catégorie d'artisanats ; on entrevoit l'intérêt de ce constat pour l'histoire économique de la cité. 


\section{Bibliographie}

\section{- Ouvrages généraux}

Ph. BRuneau, J. DuCAT, Guide de Délos ${ }^{3}$ (1983).

Ph. BRUneAU, M. BRUnet, A. FARnOUX, J.-Ch. MORETTI (dir.), Délos île sacrée et ville cosmopolite (1996).

J. CHAMONARD, Le Quartier du théâtre, EAD 8 (1922-24).

W. DEONNA, Le mobilier délien, EAD 18 (1938), passim.

W. DEONNA, La vie privée des Déliens, Travaux et Mémoires 7 (1948), principalement chapitre 5 « Métiers des Déliens. Commerce et industrie», p. 51-88, compilation toujours utile de références bibliographiques concernant les trouvailles en rapport avec l'artisanat.

\section{- Carrières}

L. CAYEUX, Description physique de l'île de Délos, EAD 4 (1911).

Ph. FRAISSE, T. KOZELJ, «Une carrière de marbre au Sud-Est du Cynthe », $B C H 115$ (1991), p. 283-296.

- Ateliers de sculpteurs

$\mathrm{Ph}$. JOCKEY, «Techniques et ateliers de sculpture à Délos à l'époque hellénistique », Nouvelles de l'archéologie 60 (été 1995), p. 11-14.

- Toreutique

G. SIEBERT, « Les arts du bronze », DossAParis 105 (mai 1986), p. 60-66.

\section{- Teintureries}

Ph. BRUNEAU, «L'industrie délienne de la pourpre », $B C H 93$ (1969), p. 759-791.

Ph. BRUNEAU, « La fabrication de la pourpre à Délos », $B C H 102$ (1978), p. 110-114.

Ph. BRUNEAU, « Encore la pourpre », $B C H 103$ (1979), p. 83-88.

Ph. BRUNEAU, « Pythagore ci-devant "pêcheur délien" », BCH 109 (1985), p. 545-546.

- Parfumeries

J.-P. BRUN, « La fabrication des parfums dans l'Antiquité », L'archéologue 20 (avril 1996), p. 35-42.

J.-P. BRUN, « Laudatissimum fuit antiquitus in Delo insula. Pressoirs et parfums à Délos à l'époque hellénistique », $B C H$ (à paraître).

- Ateliers de verriers

M.-D. NENNA, ici même p. 693-701.

- Fabricants d'auloi

A. BÉLIS, ici même p. 777-790.

\section{- Meuneries}

M. BRUNET, « Le moulin délien », in D. MEEKS, D. GARCI A (éds), Techniques et Économie antiques et médiévales : le temps de l'innovation (1997), p. 29-38.

\section{- Huileries}

J.-P. BRUN, M. BRUNET, « Une huilerie du premier siècle avant J.-C. dans le Quartier du thêâtre à Délos », $B C H 121$ (1997, à paraître).

- Pressoirs à vin

Ph. BRUNEAU et Ph. Fraisse, «Un pressoir à vin à Délos », $B C H 105$ (1981), p. 127-153.

$\mathrm{Ph}$. BRUNeAU et Ph. FraISSE, «Pressoirs déliens », $B C H 108$ (1984), p. 713-730.

Ph. BRUNEAU, « Encore les pressoirs », BCH 111 (1987), p. 339-341. 


\section{Discussion}

A. MULLER - Les remarques méthodologiques que tu as faites sont très importantes, pour toute enquête de ce genre, quel que soit le site. Le résultat que tu as obtenu est bien évidemment une incitation à en faire autant ailleurs. Tu donnes pour Délos une image des implantations artisanales qui est quand même neuve, même si elle n'était pas entièrement inconnue : pratiquement tous les éléments étaient disponibles, à l'exception des deux nouveautés que tu as développées. Certes, Délos est un cas très particulier, et l'image correspond à une période bien circonscrite dans le temps (fin $\mathrm{II}^{\mathrm{e}}$ - début $\mathrm{I}^{\mathrm{er}}$ ). On est loin en tout cas de l'image traditionnelle d'Athènes, avec le quartier des bronziers, la rue des narbriers...

M. BRUNET - Ce n'est pas la situation à Délos ! En fait, je pense qu'on a longtemps vécu sur l'image du quartier du Céramique d'Athènes, avec les potiers. C'est vrai que les potiers ont certainement parfois tendance à se grouper; les localisations s'expliquent aussi par des problèmes de nuisances. À Délos en tout cas, il est évident qu'il y avait des activités artisanales un peu partout aux abords du sanctuaire ; le seul endroit où il n'y en avait pas, en l'état actuel de nos connaissances, c'est à l'intérieur du téménos, à l'intérieur du hiéron.

M.-D. NENNA - Délos est un cas particulier, comme l'a montré M. Brunet, ne serait-ce que par l'importance de ses productions de luxe ou de semi-luxe dans une ville dominée par un sanctuaire international. On ne peut en tout cas tirer argument de l'exemple de Délos pour repousser l'image traditionnelle fournie par Athènes, avec les regroupements d'artisanats par quartier.

A. MULLER - De fait Délos est un cas spécial. Les artisanats à nuisances qui imposent des contraintes topographiques ne sont pas représentés dans le tableau dressé par M. Brunet, à l'exception des teinturiers.

V. CHANKOWSKI - La distinction que tu as faite entre produits de consommation courante, ordinaire, et produits de luxe est extrêmement intéressante. On la retrouve aussi dans le dossier épigraphique pour les prix ; c'est donc un système de fonctionnement du marché qui, finalement, n'est pas si banal que ça. En effet, les économistes - je pense en particulier à Jean-Yves Grenier qui a travaillé sur l'Ancien Régime - ont montré que cette structure du marché entre produits de consommation courante et produits de luxe était une caractéristique des marchés pré-industriels, liée à des modèles particuliers de la demande. En l'occurrence, l'épigraphie, qui fournit des exemples de prix de produits artisanaux, et l'archéologie, qui fournit les exemples d'installations artisanales, se rejoignent pour montrer que la classification des artisans, la compréhension du monde artisanal, se font en termes économiques, en termes de commerce et d'insertion sur le marché.

M. BRUNET - Tant mieux si l'on arrive à mettre en évidence l'existence de ce double circuit en quelque sorte. Mon problème était de bien faire la distinction entre une chaîne qui finalement échappe complètement au marché, qui va de l'auto-production à l'autotransformation et à l'auto-consommation, et puis le moment où l'on passe au seuil audessus. Je me demande comment arriver à saisir une catégorie qui devait être beaucoup plus importante qu'on ne le soupçonne, majoritairement domestique, mais qui dégage tout de même un petit surplus pour du troc ou un accès au marché. Or, sur ce point, l'archéologie ne fournira jamais des critères suffisants, c'est évident. Il faudrait arriver à cerner éventuellement (mais à travers quelle catégorie de documents ? - je crains qu'on ne les ait pas non plus ) ce type de situation dans les comptes.

V. CHANKOWSKI - C'est donc bien le signe qu'il existe une catégorie artisanale qui n'est pas spécifiquement, exclusivement, insérée dans le marché, mais qui se situe à la frontière des deux. 
D. VIVIERS - Quels sont les arguments archéologiques sur lesquels vous vous appuyez pour dire que l'huilerie est privée ? En effet, la même implantation, par exemple dans les villes de Syrie du Nord, qu'a très bien étudiées Georges Tate, suggère des conclusions tout à fait différentes : on considère que ces huileries sont des huileries communales, où chacun apporte ses olives ; on n'a pas ce petit surplus dont vous parliez, on ne sort pas du cadre de l'auto-production. Qu'est-ce qui indique, à Délos, que cette installation est privée et qu'on travaille dans le cadre d'un surplus économique ?

M. BRUNET - Je n'ai pas dit que c'était privé. J'ai dit que c'est un lieu où on produit 3900 litres d'huile.

D. VIVIERS - Oui, mais pas nécessairement pour une personne !

M. BRUNET - Effectivement, le problème fondamental est de savoir quel est le contexte de fonctionnement de toute cette organisation finalement rurale. C'est-à-dire de savoir quelles sont les traditions, les usages, les fonctionnements à la fois mentaux et économiques. En bref, peut-on parler de coopérative ou non ? Un élément, à Délos, me semble très important : la localisation de cette huilerie en ville. D'où viennent les olives qu'on y presse ? à mon avis, elle ne viennent pas des différentes exploitations rurales de Délos.

D. VIVIERS - Mais, dans ce cas, pourquoi ne pas importer l'huile déjà toute pressée ?

M. BRUNET - Parce que, si elle vient de Myconos, de Tinos, de pas très loin, autant fabriquer sur place. Mais il y a là un réel problème. Je suis à la recherche de tout texte pouvant m'éclairer sur la question!

R. ÉTIENNE - Est qu'au fond Délos constitue un modèle urbain particulier ou non ? Il faudrait voir comment s'insère l'artisanat dans d'autres villes fouillées extensivement, comme Olynthe ou Priène : dispose-t-on de données suffisantes ? En tout cas, on dispose, à Pella, d'un tout autre modèle urbain : une grande concentration d'ateliers-magasins, installations de fabrication et de vente, autour de l'agora. Avons-nous à faire ici à un système de fonctionnement socio-économique différent, ou simplement à une autre conception de la ville, avec le regroupement topographique des activités artisanales ? Je n'en sais rien, mais je crois qu'il faut bien poser les questions et opposer des modèles de distribution que l'on commence quand même à cerner. 